\title{
Reliable Data Collection in Wireless Sensor Networks
}

\author{
Pramod A. Dharmadhikari \\ PG Student \\ M B E Society's College of \\ Engineering, Ambajogai \\ Maharashtra, India
}

\author{
B. M. Patil \\ Professor \\ M B E Society's College of \\ Engineering, Ambajogai \\ Maharashtra, India
}

\author{
V. M. Chandode \\ Associate Professor \\ M B E Society's College of \\ Engineering, Ambajogai \\ Maharashtra, India
}

\begin{abstract}
In wireless sensor networks the nodes which are near to the sink node operate as intermediate node. The source nodes will generate events as per the frequency provided to them and forward packets to the sink through intermediate nodes. The sink node could not able to collect data packets reliably since excessive number of packets drop at intermediate nodes in congestion situations. An efficient storage mechanism needed to store the packets as well to diminish packet loss. The objective of the current work is to enhance reliable data collection at sink node by using intermediate storage nodes to alleviate congestion problem in wireless sensor network. In this paper we present a typical database file attached model in vicinity to data packets storage at cluster head nodes. An attached storage database file to cluster head node will advantageous in storing data packets dropped during transmission. The dropped packets are recollected by the cluster head node from attached database file on getting negative acknowledgement from sink. The recovered data packets sent back to the sink node which will diminish packet loss. Consequently, reliable data collection is achieved at sink node.
\end{abstract}

\section{General Terms}

Data collection in wireless sensor networks, data storage in wireless sensor networks.

\section{Keywords}

WSN, Wireless Sensor Networks, PDR, Reliability.

\section{INTRODUCTION}

Wireless Sensor Networks (WSNs) have recently emerged as a new area in networking research. WSN is a collection of one or more sensor nodes. The sensors are battery operated low power consuming devices having components like processor, memory, power supply, radio transceiver, and analog to digital converter [1]. There may also present several hundreds or even thousands of sensor nodes in the network. These small sensor nodes have ability to communicate with each other wirelessly. The Different kinds of sensors may be attached in the sensor network to measure different physical and environmental parameters. The sensor nodes are used to detect, measure, and record physical event/properties like temperature, moisture, motion, pressure, vibration, sound, light, radiation, image, chemical changes etc. Usually, sensor nodes senses physical data and transmit the gathered information to base station or sink node [2].

The applications of wireless sensor network can be categories as remote environmental monitoring and target tracking. The monitoring applications includes physical or environmental event monitoring at indoor/outdoor locations, factory monitoring, process automation monitoring, health and wellness monitoring, power monitoring, inventory monitoring, structural monitoring etc. The tracking applications include military tracking applications, objects tracking, animals tracking, humans tracking, and vehicles tracking etc [2].

There are many problems to be solved in wireless sensor network, such as congestion control, rate control, flow control, medium access control, queue management, power control and topology control etc [3].

In a Wireless sensor network a large amount of data flows from sensors to sink. When large numbers of sensor nodes are transmitting the data packets, the load becomes heavy and data traffic also increases and this might lead to congestion situation. Congestion in a wireless sensor network may leads to problems like data packet loss, delay of critical information, wastage of resources, buffer overflow. In wireless sensor network application packet loss occurs at different levels. The packet loss may occur due to overflow of buffer capacity, congestion situation, packet collision, poor radio communication, and failure of node. The packet loss results in wasted energy and degraded quality of service [3].

In a wireless sensor networks one common critical service is data collection, where sensed data are continuously collected by sensor nodes and forwarded to a central base station for further processing. The detection of packet loss and correctly recovering missing packets is important factor to be considered. Wireless sensor networks need to be overcome problems like congestion control, reliable data collection or dissemination, energy conservation etc. Some mechanism should be implemented to avoid data packet loss in congestion situations.

The current work concentrates on improving data collection at sink node. It focuses data packets storage mechanism as primary task in a wireless sensor network to achieve reliable data collection in wireless sensor network. The packet loss is minimized by retransmitting the dropped packets to sink. The dropped packets are recovered from attached database file. This technique improves data collection by avoiding congestion situations in the wireless sensor network.

\subsection{Characteristics of wireless sensor networks}

The wireless sensor network system should have following characteristics: fault tolerant, scalable, long life, programmable, secure, and affordable.

- Fault tolerant: the system should be robust against node failure (running out of energy, physical destruction, hardware, software issues etc). Some mechanism should be incorporated to indicate that the node is not functioning properly. 
- Scalable: the system should support large number of sensor nodes to cater for different applications.

- Long life: the sensor node should be power efficient. It is difficult to replace or recharge thousands of nodes. The node's communication, computing, sensing and actuating operations should be energy efficient too.

- Programmable: the reprogramming of sensor nodes in the field might be necessary to improve flexibility.

- Secure: the system should be secure enough to prevent unauthorized access to node information

- Affordable: the system should use low cost devices since the network comprises of thousand of sensor nodes, tags and apparatus. Installation and maintenance of system elements should also be significantly low to make its deployment realistic.

\subsection{Protocol stack of wireless sensor networks}

The protocol stack of wireless sensor network consists of layers application layer, transport layer, network layer, MAC layer, physical layer. The multiple applications can be run on the application layer with respect to different sensing operations. The transport layer regulates the flow of data, congestion situation, avoids packet loss, ensure the reliability and quality of data at the source and the sink. The network layer performs the routing of the data supplied by the transport layer. The MAC layer manages power utilization of sensor nodes in different environments like is noisy sensor nodes environment, mobile sensor node environment, the MAC protocol must be power aware and able to minimize collision with neighbor's broadcast. The physical layer takes care of a modulation techniques, transmission and receiving techniques [1]

\section{LITERATURE SURVEY}

Akan et al. presented a new reliable transport scheme for wireless sensor network, the ESRT (Event-Sink Reliable Transport) protocol [4]. ESRT is an end-to-end technique. The ESRT protocol achieves reliability and also conserves energy by help of congestion control component. The guarantee of reliability is given by regulating sensor report frequency.

PSFQ: Pump Slowly Fetch Quickly [5] is a simple transport protocol. It uses hop-by-hop error recovery technique. In this case intermediate nodes also perform loss detection and recovery. In this protocol the data distributed from a source node with slow speed (pump slowly), but the nodes which finds data loss are allowed to fetch (i.e. recover) any missing data from immediate neighbors quickly.

Growth codes [6] are the techniques to increase "persistence" of sensed data. The data is replicated in compact form at neighboring nodes using "Growth Codes" which will increase efficiency of data accumulation at the sink node.

An energy efficient data storage policy for object tracking wireless sensor network explained in [7]. A local storage method with small control overhead is proposed for object tracking application in wireless sensor network. The detected events are stored in local storage and the relation of the sensed data maintain through a linked list among sensor nodes.

Khan et al. presents in-network storage model for data persistence under congestion in wireless sensor network [8].This model is built with dense clustered sensor field. Here, the routing nodes act as data buffers during congestion periods in order to avoid data loss. The storage model can be used in combination with congestion avoidance/control techniques.

A data storage method based on query processing explained in [9]. The main storage methods categorized as Exterior Storage (ES), Local Storage (LS), and Data-Centre Storage (DCS).

A reliable transport protocol proposed for wireless sensor networks in [10]. The model uses the hop-by-hop technique. Each intermediate node has two types of buffer, the receive buffer and retransmission buffer respectively. All the received packets are placed in the receive buffer also copy of each received packet is saved in a cache memory. When a node receives the ACK of its already sent packet, it removes the packet from its local cache. Packets which are received with negative ACK are forwarded to the retransmission buffer.

The reliability of sensor network explained in [11, 12]. The node senses the physical or environmental parameters and forwards the corresponding data to the sink through cluster head. The energy consumption of the nodes near sink will always greater, as these nodes are continuously busy with data packet routing to sink. The nodes which are at longer distance from the cluster head or sink are having lower energy consumption as compare with the nodes near the cluster heads or sink.

An idea of enhancing reliability on wireless sensor network by AODV-ER routing protocol implemented in [13]. The proposed protocol finds the route which has the highest end-to-end reliability from the source to the destination based on the hop-by-hop packet reception probability.

A reliability model for extending cluster lifetime using backup cluster heads proposed in [14]. In the cluster-based two-tier wireless sensor networks, the cluster-head nodes gather data from sensors and then transmit to the base station. When the cluster head dies, backup cluster head takes over the responsibility and continues to work as a new cluster head.

Sheng et al. address the optimal storage node placement problem in [15]. The storage nodes are available to store the data collected from the sensors. The storage nodes alleviate the heavy load of transmitting all data to a central place for storage. The model also reduces the query communication cost.

Prabakaran et.al, described queue reloading scheme for congestion leveling in wireless sensor networks [16]. The proposed queue reloading scheme controls the node level congestion. In order to avoid the retransmission of packets, the proposed scheme uses an alternative queue for caching the overflowed packets. The dropped packets are reloaded from the alternative queue when the current queue becomes available.

\section{PROPOSED WORK}

In wireless sensor networks sensor nodes senses physical parameters data and transmit the gathered information to base station. The source node will generate events as per the frequency provided to that source node. If all source nodes forward there packets towards the sink the possibility of congestion is more since each node will process these packets in hop by hop data dissemination. The nodes near to the sink operate as intermediate node, there energy will consume more so, they dead early and further data packets transmission from source nodes to sink clogged that leads to congestion situation in network. Congestion in wireless sensor network may leads to higher packets drop, lower throughput and increased delay. 
To evade packet loss persistent data storage needed at intermediate nodes, so that sink can retrieve the dropped packets from stored database file.

We proposed two architectures for measuring reliability in data collection. The first architecture is hierarchical cluster based architecture is shown in Figure 1. In this architecture a cluster of few nodes formed in hierarchical manner all source nodes in that cluster will sense parameter data values and forward data packets towards the sink through their respective cluster head node.

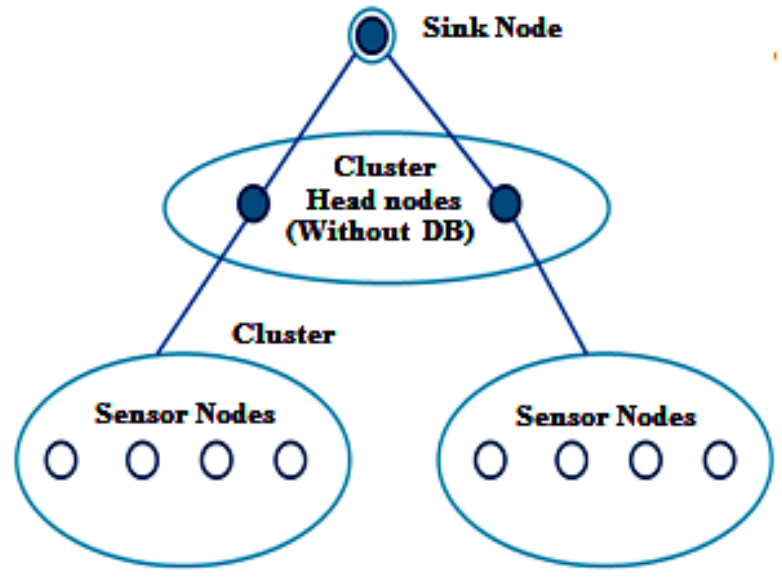

Figure 1: Hierarchical cluster based architecture (Cluster head without database)

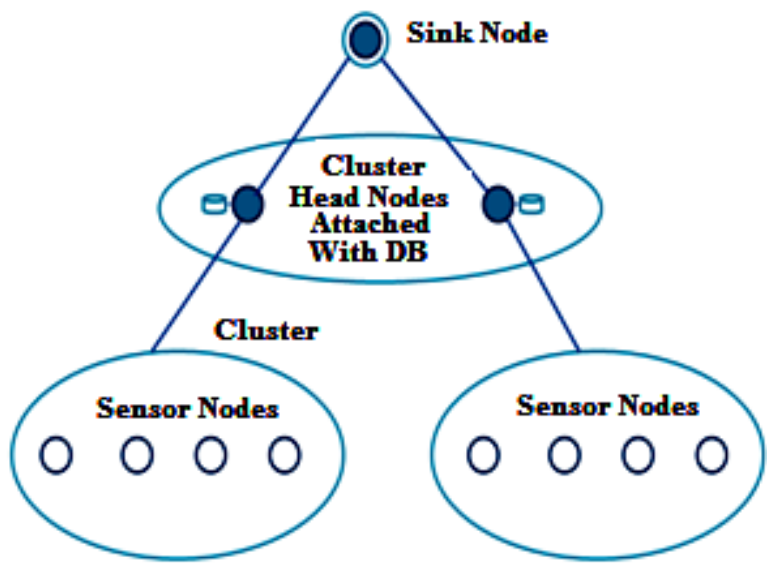

Figure 2: Hierarchical overlay based architecture (cluster head as storage node attached with database file)

The second architecture is hierarchical overlay based architecture is shown in Figure 2. This architecture is similar as first hierarchical cluster based architecture, in addition to that, each cluster head act as storage node that attached with database file. The source nodes forward sensed data packets towards their cluster head which will further transmits packets to the sink. The dropped data packets are stored in database file attached to cluster head automatically in background. The packets which are dropped in between cluster head and sink during transmission will be identified by cluster head on getting negative acknowledgement from sink node. These packets will be sent back to the sink by cluster head node after recovering dropped packets from database file. So, packet loss is minimized and reliable data collection can be achieved at sink node.

\section{IMPLEMENTATION}

We have implemented both architecture models using NS-2 based simulation, both architecture models looks same but in case of hierarchical overlay based model database file is attached to cluster head nodes. The packets storage work will perform automatically in background. The actual implementation of both architecture using NS-2 simulations looks same as shown in Figure 3 but in case of hierarchical overlay based second architecture we have attached database file to the cluster head nodes. The details of both architectures are explained below.

\subsection{Hierarchical cluster based architecture (cluster head without database file)}

The ns-2 simulation implemented architecture is shown in Figure 3. Initially, the source node will generate events as per the frequency provided to that source node. All source nodes forward there packets towards to the sink (node 0) through cluster head (node 1\&2). Here in this case no database file attached to cluster head node. Cluster head only performs normal packet transmission from source node to sink. Due to limited or insufficient buffer size at cluster node there is more packets drop while transmission of packets from source node to sink. So, this architecture suffers from the lower packet delivery ratio. To overcome the packet drop issues we implemented another model i.e. overlay based hierarchical model where we have attached database file to cluster head nodes. This model results with lower packet delivery ratio, more end-to-end delay, more packet drop, less received packets compare with overlay based architecture where database attached to the cluster head.

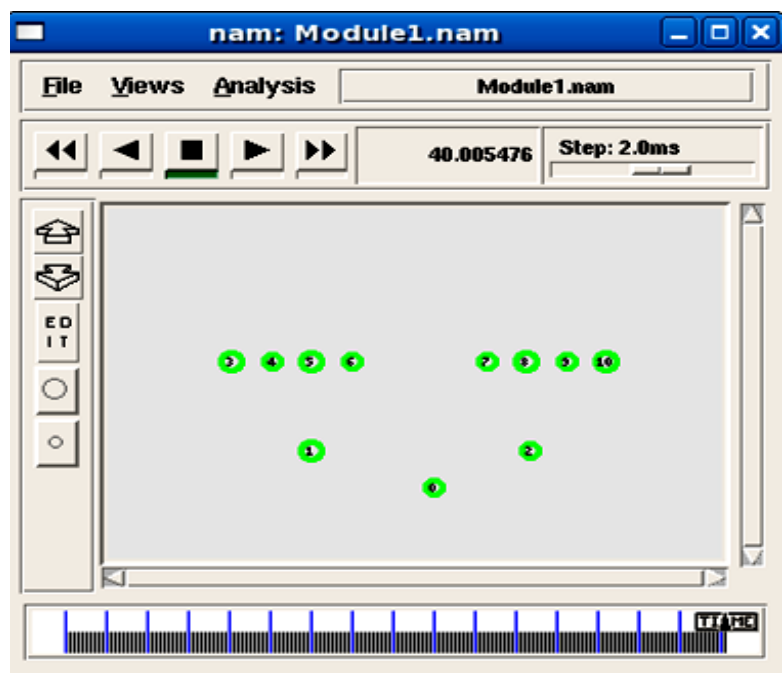

Figure 3: NS-2 simulations for both hierarchical architectures

\subsection{Hierarchical overlay architecture (cluster head attached with database file)}

The ns-2 simulation implemented architecture is shown in Figure 3. This architecture model looks same as cluster based architecture in simulation but attached with database file in background. Initially, the source node will generate events as per the frequency provided to that source node. All source nodes forward there packets towards to the sink through cluster head. Here in this case database file attached to cluster head node. The cluster head (node $1 \& 2$ ) collects incoming data packets from source node and simultaneously forwards it to sink (node 0 ). The packets which are discarded or dropped 
while transmission from cluster head node to sink node will be pushed into attached database file automatically. When cluster head will get negative acknowledgement from sink node, it will recollect drop packets from database file and resent those data packets to the sink node. Hence, by using this architecture we reduce the congestion and increase the reliable data collection at sink node.

In NS-2 we can create simulation using number of nodes with variety of topologies. Each node has associated with queue of user defined length to store data packets (IFQ length value). During the transmission of packets when this queue full with adequate number of packets further incoming packets will be dropped as no sufficient space. The dropped packets could not be easily recovered. A certain data packet recovery mechanism needed, we have implemented that using a database file. The database file is created using C++ and TCL scripting. The packets which are dropped while transmission from cluster head node to sink will be marked as discard. All the discarded packets are automatically push into database file. When packet drops the negative acknowledgement will be received by cluster head node from sink. The cluster head node then recollects dropped packet from attached database file and retransmits data packets to sink node. The database file is dynamic in size, it can accommodate as many as numbers of packets. This architecture model results with improved packet delivery ratio, more number of received packets, less number of dropped packets as well as minimized end-to-end delay compare with previous hierarchical cluster based architecture. The overhead on architecture is energy consumption issues of nodes. The energy consumption is greater in hierarchical overlay based database attached model because of frequent storage and retransmissions for dropped packets. The simulation parameters for implementation are given in Table I below.

Table 1 Ns-2 Simulation Parameters

\begin{tabular}{|l|l|}
\hline Area of sensor field & $1000 * 1000 \mathrm{~m}$ \\
\hline Topology used & Hierarchical \\
\hline Packet length & 512 Bytes \\
\hline IFQ length & 100 Packets \\
\hline Transmission range & $200 \mathrm{~m}$ \\
\hline Interference range & $550 \mathrm{~m}$ \\
\hline Initial Energy & 100 Joules \\
\hline MAC layer protocol & 802.11 \\
\hline Routing protocol & AODV \\
\hline Frequency of packet generation & $(1 /$ Time interval $)$ \\
\hline Number of sensor nodes & 11 Nodes \\
\hline Source nodes numbers & $4,5,8,9$ \\
\hline Cluster head numbers & 1,2 \\
\hline Sink node number & 0 \\
\hline
\end{tabular}

\section{PERFORMANCE ANALYSIS}

In performance analysis we have compared above two models i.e. hierarchical cluster based architecture based architecture (cluster head without database) and overlay based model (cluster head attached with database). For performance analysis we have considered packet size as 512 bytes, reporting rate (1/interval time) of 10 , routing protocol AODV, MAC protocol 802.11 and initial energy of 100 Joules. We compare these two models on basis of packet delivery ratio, received packets, drop packets, end-to-end delay. The graphs shown below illustrate comparative analysis of both models. Overall the hierarchical overlay based model (database attached model) will give better results.

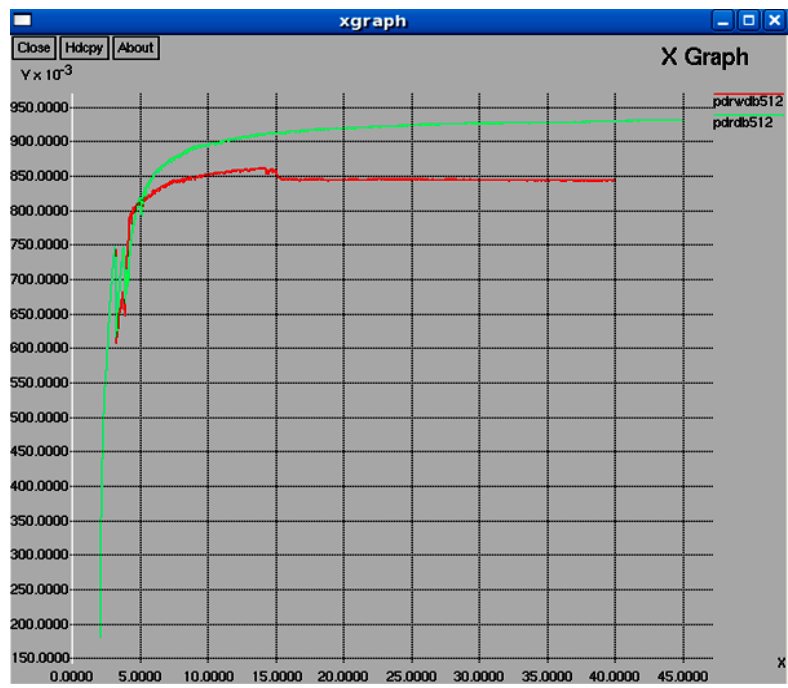

Figure 4: Comparative graph for packet delivery ratio

The graph shown in Figure 4 illustrates that, PDR (Packet Delivery Ratio) for hierarchical overlay based database attached model is high as compare to hierarchical cluster based without database attached model. In database attached model packets are resent to the sink by cluster head nodes from attached database file hence, packet delivery ratio will increases. Here Reporting Rate (RR) is 10 and packet size is of 512 bytes. The $\mathrm{X}$-axis represents reporting rate (i.e. 1/interval time) and Y-axis represents PDR (Packet Delivery Ratio) value 0.1 to 1.0 (highest PDR value). The graph lines uses naming convention as "pdrwdb512" (packet delivery ratio without database, packet size 512 Bytes) and "pdrdb512" (packet delivery ratio, database, packet size 512 Bytes).

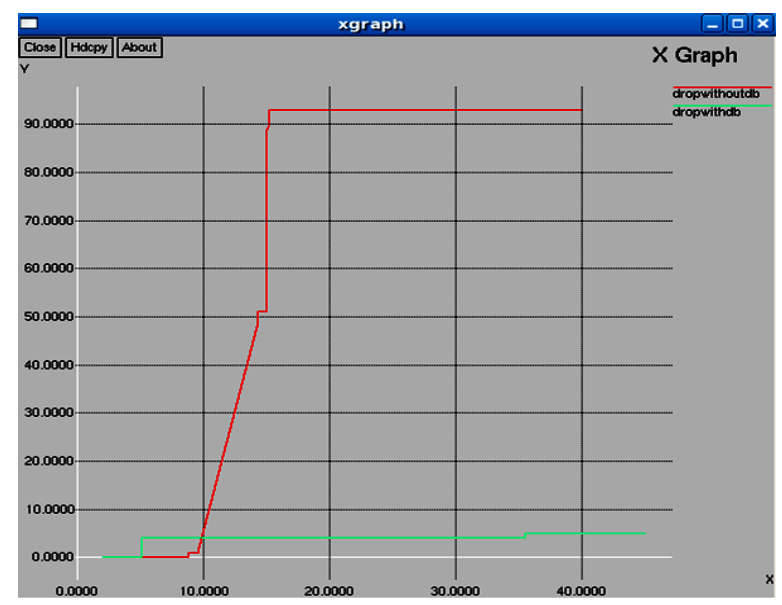

Figure 5: Comparative graph for packets drop

The graph in Figure 5 shows that the packet drops for hierarchical overlay based database attached model is less as compare to hierarchical cluster based without database attached model. In hierarchical overlay based database attached model packets are retransmitted to the sink by cluster head node from attached database file. Here drop packets recovered from database file and resent to sink node. The graph lines uses naming convention as "dropwithoutdb" (packet drop without database attached model), and "dropwithdb" (packet drop with database attached model). 
Here reporting rate is of 10 which represented on $\mathrm{X}$-axis and Y-axis represents represents packets drop values.

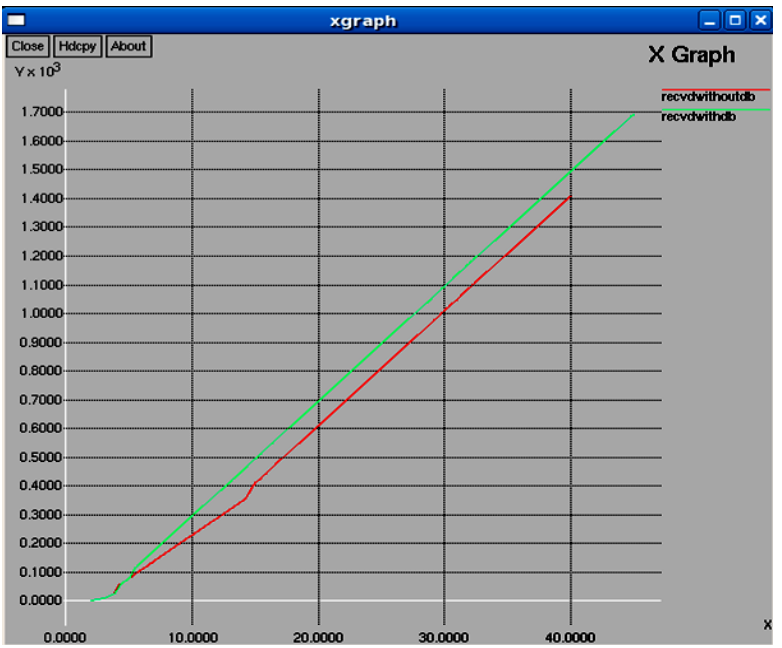

Figure 6: Comparative graph for packets received

The packets received for hierarchical overlay based database attached model are superior as compare to hierarchical cluster based without database attached model as shown in Figure 6 . Here reporting rate is 10 and packet size is of 512 bytes. The $\mathrm{X}$-axis represents reporting rate and $\mathrm{Y}$-axis represents packets received values. The graph lines uses naming convention as "recvdwithoutdb" (received without database attached model), and "recvdwithdb" (received with database attached model). In hierarchical overlay based database attached model packets drop packets recovered from database file and resent to sink node by cluster head node which will increases more number of received packets.

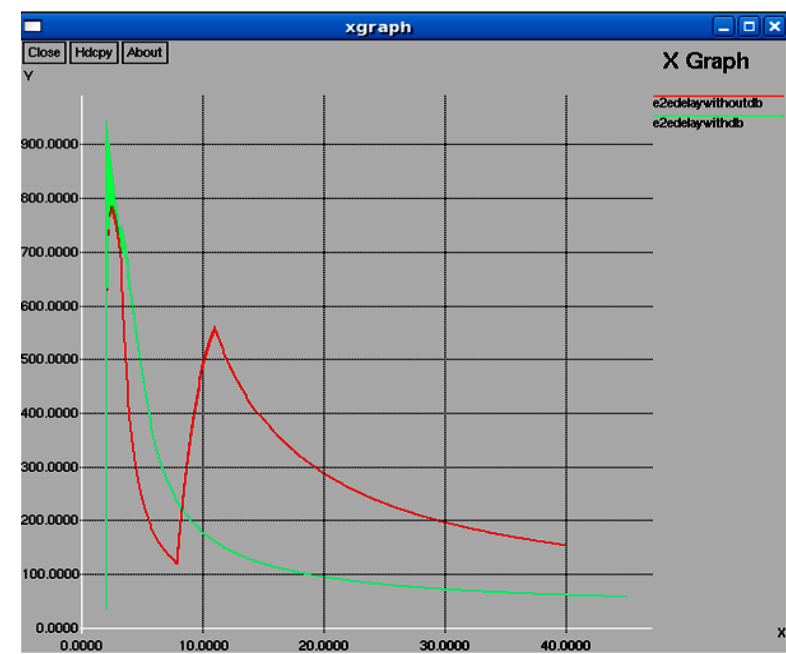

Figure 7: Comparative graph for end-to-end delay

The graph shown in Figure 7 demonstrates end-to-end delay comparison traced with reporting rate of 10 and packet size of 512 bytes. Here $\mathrm{X}$-axis represents reporting rate and $\mathrm{Y}$-axis represents delay values in milliseconds. Initially end-to-end delay of both architectures increases and later it will decreases. Comparatively in with database attached model end-to-end delay is constantly less than without database attached model after reporting rate value 10 . The end-to-end delay increases due to congestion situation in network. The graph lines uses naming convention as "e2edelaywithoutdb" (end-to-end delay without database attached model), and "e2edelaywithdb" (end-to-end delay with database attached model).

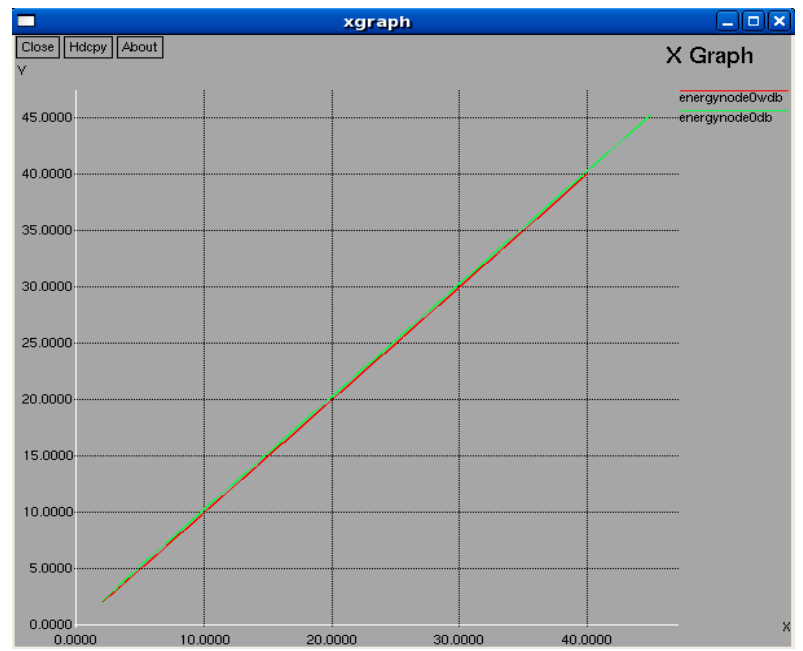

Figure 8: Comparative graph for energy of sink node0

The graph shown in Figure 8 traced out initial energy of 100 Joules, with reporting rate 10 and packet size of 512 bytes. It indicates energy consumption of sink node 0 for hierarchical models with database attached and without database attached models. The energy consumed by sink (node 0 ) in case of hierarchical overlay based with database attached model is slightly more than hierarchical cluster based without database attached model. The initial energy value of node is taken as 100 Joules. The $\mathrm{X}$-axis represent reporting rate and $\mathrm{Y}$-axis represent energy consumption values in Joules. The graph lines uses naming convention as "energynode $0 \mathrm{wdb}$ " (energy of sink node 0 , without database model indicated by red line) and "energynode0db" (energy of sink node 0 , database model, indicated by green line).

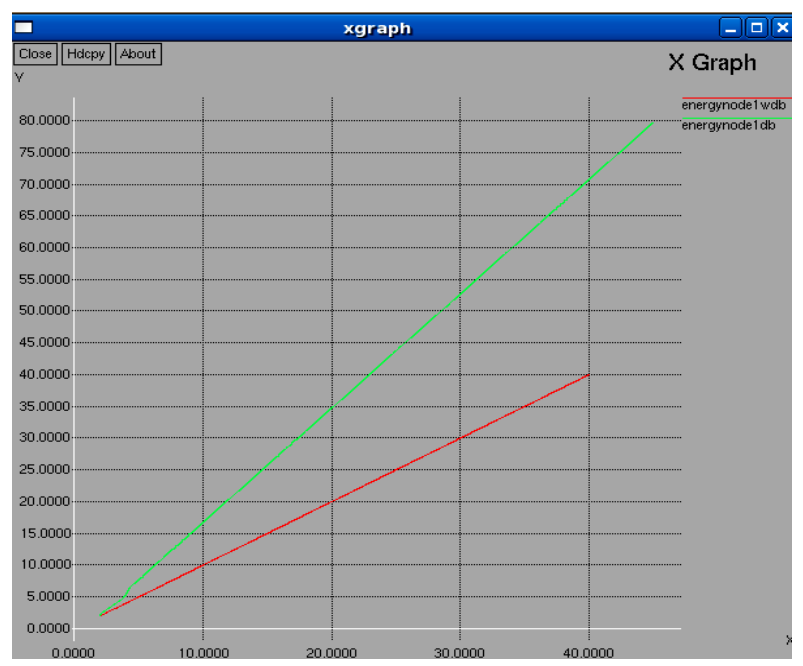

Figure 9: Comparative graph for energy of cluster head node1

The graph shown in Figure 9 outlines energy consumption of cluster head node 1 for hierarchical models with database attached and without database. The energy consumed by cluster head node 1 in case of with database attached model is greater than without database model.

The cluster head node 1 in database attached model consumes more energy due to additional burden of data packet collection, packet storage and number of retransmission for 
sending dropped packets to sink node. The graph lines uses naming convention as "energynodelwdb" (energy of cluster head node 1, without database model indicated by red line) and "energynodeldb" (energy of cluster head node 1, database model indicated by green line). Here, the X-axis represent reporting rate and $\mathrm{Y}$-axis represent energy consumption values in Joules. The reporting rate is 10 , packet size is of 512 bytes and initial energy is of 100 Joules.

\section{CONCLUSIONS \& FUTURE WORK}

The data which flows through the wireless sensor network has great impact on the link load collecting data reliably at sink node against the congestion issues is a foremost task. The packets loss recovery and reliability are the leading challenges in wireless sensor network. A proficient data packets storage mechanism needed to inhibit packets loss. In our hierarchical overlay based architecture model we have attached database file to cluster head node for storage of data packets. The data packets which are lost during transmission from cluster head node to sink node marked as discard and those discarded packets automatically pushed in to database file. On getting negative acknowledgement from sink to cluster head node the dropped packets will be recollected from database file and sent back to sink node by cluster head node providing way for reliable data collection at sink node. Our ns-2 simulation results graph analysis shows that hierarchical overlay based database attached data collection model will provide the improved packet delivery ratio, more number of received packets, less number of dropped packets and lesser end-to-end delay.

The hierarchical overlay based database attached architecture model minimizes the congestion and improves the reliability of network in data collection at sink node. Some of the directions for future work are as follows:

1) If the cluster head node dead early then will it possible to transfer packets through other cluster head.

2) Energy consumption issues are needed to be improved.

\section{REFERENCES}

[1] I. Akyildiz, W. Su, Y. Sankarasubramaniam, and E. Cayirci, "A survey on sensor networks," Elsevier Journal in computer networks, vol. 38, no.4, pp.393-422, 2002.

[2] J. Yick, B. Mukherjee, and D. Ghosal, "Wireless sensor network survey," Elsevier Journal in computer networks, vol. 52, no.1, pp.2292-2330, 2008.

[3] C. Wan, S. Eisenman, and A. Campbell, "CODA: Congestion Detection and Avoidance in sensor networks," Sensys in proceedings of conference on wireless network, NewYork,, USA, vol. 5, no. 7, 2004.

[4] O. B. Akan, and I. Akyildiz, "Event-to-Sink Reliable Transport in wireless sensor networks," IEEE/ACM Transactions on networking, vol. 13, no. 5, 2005.

[5] C. Wan, A. Campbell, and L. Krishnamurthy, "Pump-Slowly Fetch-Quickly (PSFQ): A reliable transport protocol for sensor networks," IEEE Journal on selected areas in communications, vol. 23, no. 4, 2005.
[6] A. Kamra and V. Misra, "Growth Codes: maximizing sensor network data persistence," IEEE in proceedings of international conference, 2006.

[7] S. Ho and G. Yu, "An energy efficient data storage policy for object tracking wireless sensor network," IEEE, proceedings of the IEEE international conference on sensor networks, ubiquitous, and trustworthy computing, 2006.

[8] M. Khan, W. Gansterer, and G. Haring, "In network storage model for data persistence under congestion in wireless sensor network," IEEE in proceedings of the first international conference on complex, intelligent and software intensive systems, pp.221-228, 2007.

[9] F. Li, C. Li, X. Yang, and G. Yu, "A data storage method based on query processing region in wireless sensor networks" IEEE in proceedings of ninth international conference for young computer scientists, pp. 1367-1372, 2008 .

[10] M. Yaghmaee and D. Adjeroh, "Reliable transport protocol for wireless sensor networks," IEEE in proceedings of international symposium on telecommunications, pp. 440-445, 2008.

[11] V. Deshpande, P. Sarode, and S. Sarode, "Root cause analysis of congestion in wireless sensor networks," International Journal of Computer Applications, vol.1, no. $18,2010$.

[12] J. Helonde, V. Wadhai, V. Deshpande, S. Sutar, "EDCAM: Early Detection Congestion Avoidance Mechanism for wireless sensor network," International Journal of Computer Applications, vol. 7, no.2, 2010.

[13] N. Phong and M. Kim, "Enhancing reliability on wireless sensor network by AODV-ER routing protocol," IEEE in proceedings of second international conference on computer engineering and technology, vol.6, pp. 32-36, 2010.

[14] S. Hashmi, M. Rahman, H. Mouftah and N. Georganas, "Reliability model for extending cluster lifetime using backup cluster heads in cluster-based wireless sensor networks," IEEE in proceedings of first international workshop on advances in wireless and actuator networks, pp. 479-485, 2010.

[15] B. Sheng, Q. Li, and W. Mao, "Optimize storage placement in sensor networks," IEEE transactions on mobile computing, vol.9, no.10, 2010.

[16] N. Prabakaran, B. Shanmugaraja, and R. Prabakaran, "Queue reloading scheme for congestion leveling in wireless sensor networks," IEEE in proceedings of international conference on devices and communications, pp.1-5, 2011

[17] N. Prabakaran, B. Shanmuga, R. Prabakaran, and V. Sarma Dhulipala, "Rate optimization scheme for node level congestion in wireless sensor networks," IEEE in proceedings of international conference on devices and communication, pp.1-5, 2011 